\title{
Vergleichende Untersuchungen über die Ausscheidung von Neosalvarsan, Sulfoxylatsalvarsan und Silbersalvarsan durch die Niere und die Beziehungen zur „Depotwirkung““.
}

\author{
Von \\ Dr. med. Eugen Bernstein.
}

(Aus der Universitäts-Hautklinik Frankfurt a. M. [Direktor: Geh. Med.-Rat Prof. Dr. K. Herxheimer] und der chemischen Abteilung [Vorstand: Prof. Dr. A. Binz] des Georg Speyer-Hauses [Direktor: Geh. Med.-Rat Prof. Dr. Kolle].)

Mit 7 Textabbildungen.

(Eingegangen am 11. Februar 1921.)

Nachdem Paul Ehrlich das Salvarsan in die Syphilistherapie eingeführt hatte, richteten sich naturgemäß die Bestrebungen darauf, das Salvarsan in chemischer und biologischer Beziehung zu verbessern. Insbesondere kamen hierbei diejenigen Eigenschaften des Salvarsans, die für den Praktiker unbequem waren, nämlich das umständliche Lösungsverfahren und die Unbeständigkeit der gelösten Verbindungen an der Luft in Frage. Sowohl das Neosalvarsan wie das Salvarsannatrium erfüllten die ersten Forderungen nur teilweise. Auch auf die Verbesserung des chemotherapeutischen Index waren die Bestrebungen gerichtet. Die von W. Kolle nenerdings eingeführten Präparate, das Silbersalvarsan und Sulfoxylatsalvarsan bedeuten eine weitere Etappe auf diesem Weg.

$\mathrm{Zu}$ den aktuellsten Fragen der Anwendung der einzelnen Präparate gehört meines Erachtens die sogenannte „Indikationsdifferenzierung" derselben. Das erstrebenswerte Ziel bei der Behandlung der primären und der sekundären Lues ist die Darstellung eines Mittels, das die Treponemen rasch wegfegt. Dagegen wird man für die Nachbehandlung der Lues sowie für die Therapie syphilitischer Veränderungen der inneren Organe, wo es kaum gelingen dürfte, die im schlecht durchbluteten Gewebe versteckten Erreger mit einem Schlage abzutöten, nach treponemociden Medikamenten zu suchen haben, die nicht nur kurze Zeit in den Säften des infizierten Organismus in wirksamer Form enthalten sind, bei welchen vielmehr der Körper womöglich längere Zeit hindurch unter dem Einflusse des Präparates in milderer Form gehalten wird. Dies kann dadurch erzielt werden, daß ein Arsenobenzol- 
derivat in Depots, ähnlich wie bei der Quecksilbertherapie, abgelagert wird, aus denen es dauernd in den Kreislauf übergeht. Das Setzen von intramuskulären Depots hat aber, wie es im Anfang der Salvarsanära. üblich war, wie auch aus der Quecksilber-Depotbehandlung bekannt. ist, neben großen Vorteilen auch erhebliche Nachteile. Die Resorption ist oft ungenügend, die Depots erfahren Abkapselungen dureh entzündliche Vorgänge, wodurch nicht selten eine verzögerte oder schubweise und unregelmäßige Resorption bedingt wird.

Zwar wurde infolge der der intramuskulären Injektionsweise anhaftenden Mängel (Infiltrate, Nekrosen) diese Anwendungsart verlassen, es muß jedoch zugegeben werden, daß dieses Verfahren hinsichtlich der therapeutischen Wirkung der intravenösen Applikationsweise, infolge der langsamen Elimination des Präparates aus dem Körper, vielfach als überlegen betrachtet wird. Man suchte daher bei der intravenösen Injektion beschleunigte Ausscheidung durch multiple Salvarsaninjektionen zu ersetzen. Anderseits konnte jedoch durch die Arbeiten vieler Autoren festgestellt werden, daß auch bei intravenöser Anwendung Salvarsandepots in der Leber, Milz, Niere, Lunge, Herz, Knochen, Muskeln, Gehirn usw, gesetzt werden.

Unter Berücksichtigung dieser Gesichtspunkte wurden die neuen Arsenobenzolpräparate, das Silbersalvarsan und Sulfoxylat neben dem Neosalvarsan in den Kreis der Untersuchung gezogen, um zu sehen, ob sich aus den Ausscheidungskurven Anhaltspunkte für eine Gesetzmäßigkeit der im Vergleich mit dem Neosalvarsan. gesteigerten Wirkung dieser Präparate ergeben. Es ließ sich z. B. auf diese Weise feststellen, ob die rasche oder langsame oder nachhaltige Wirkung eines Präparates mit den Ausscheidungs- bzw. mit dem damit im Zusammenhang stehenden Kreisen des Präparates in Blut und Körpersäften in Beziehung steht. Außerdem bot aber die vergleichende Untersuchung der Arsenausscheidung bei mehreren gleichartig behandelten Patienten eine erwünschte Gelegenheit für die vergleichende Prüfung der Nierenfunktion, denn wir wissen ja, daß die Niere als. geschädigtes Organ in vielen Publikationen über Salvarsan eine besondere Rolle spielt.

Durch die Arbeiten zahlreicher Autoren (Literatur siehe bei Meirowsky und Kretschmer, Ergebnisse der Haut- und Geschlechtskrankheiten, 3. Jg. 1914, S. 533) wurde gezeigt, daß die Arsenobenzolderivate den Körper, dem sie intravenös oder intramuskulär einverleibt wurden, vor allen Dingen durch die Niere, weiterhin aber auch noch durch die $\mathrm{Haut}$, den Darm, Galle, Speichel usw. mehr oder weniger rasch verlassen. Um die Ausscheidungsverhältnisse der verschiedenen Salvarsanpräparate und damit eventuell ihre Depotwirkung exakterweise analysieren zu können, müßte es daher eigent- 
lich wünschenswert sein, alle in Betracht kommenden Ex- und Sekrete in möglichst geringen, regelmäßigen Zeitabständen einer genauen chemischen Untersuchung auf ihren Arsengehalt zu unterziehen. Da dies aber praktisch nicht durchführbar ist, beschränkte ich mich darauf, lediglich den Verlauf der Arsenausscheidung mit dem Urin zu verfolgen und aus derart erhaltenen Ausscheidungskurven auf das Verhalten der Salvarsanpräparate im Organismus und die Schnelligkeit, mit der sie denselben verlassen, Schlüsse zu ziehen.

Während sich bisher die Autoren darauf beschränkten, die Ausscheidung des Salvarsans in der Tagesmenge des Urins zu untersuchen, schien es mir zur Klärung des Problems von Wichtigkeit, in relativ ganz kurzen Zeitabständen (3 Stunden) quantitativ die ausgeschiedene Arsenmenge festzustellen ${ }^{1}$ ).

Der zu untersuchende Urin wurde zur Trockene eingedampft und die organische Substanz nach dem Verfahren von Lockeman $n^{2}$ ) durch Schmelzen mit einem Gemisch von Kalium- und Natriumnitrat zerstört. Die Bestimmung des Arsens geschah nach der kolorimetrischen Methode von $\left.\mathrm{S}_{\mathrm{mith}}{ }^{3}\right)$, die in der von Beck und Merres ${ }^{4}$ ) beschriebenen Weise ausgeführt wurde. Nach diesem Verfahren wird das zu bestimmende Arsen, das in mineralisierter Form vorliegen muB, mit Zink- und Schwefelsäure in Arsenwasserstoff übergeführt und dieser auf einen Streifen von Quecksilberbromid absorbiert. Hierbei entsteht eine orangefarbene Additionsverbindung von Arsenwasserstoff und Quecksilberbromid, die den Streifen (s. S. 58) auf eine gewisse Länge anfärbt. Die Länge des Farbstreifens ist der Arsenmenge proportional. Der Vorgang soll nach Got the $1 f^{5}$ ) nach folgenden Gleichungen verlaufen:

$$
\begin{aligned}
& \mathrm{AsH}_{3}+2 \mathrm{HgCl}_{2}=\mathrm{AsH}(\mathrm{HgCl})_{2}+2 \mathrm{HCl} \\
& \mathrm{AsH}_{3}+3 \mathrm{HgCl}_{2}=\mathrm{As}(\mathrm{HgCl})_{3}+3 \mathrm{HCl} .
\end{aligned}
$$

Zur Erzielung gleichmäßiger Resultate ist eine gleichmäßige Wasserstoffentwicklung unerläßlich. Deshalb wurde stets mit gleichen Mengen Zink- und Schwefelsäure gearbeitet. Die nach dem Mineralisieren des Harns resultierende Schwefelsäurelösung, die wechselnde Mengen Schwefelsäure enthält, wurde deshalb erst neutralisiert und dann wieder mit $100 \mathrm{ccm}$ Normal-Sehwefelsäure versetzt.

Der Arbeitsvorgang gestaltete sich folgendermaßen: Der Harn $(100-250 \mathrm{ccm})$ wurde in eine Porzellanschale auf dem Wasserbade nach Zusatz von $10 \mathrm{ccm}$ rauchender Salpetersäure zur Trockne eingedampft. Der Rückstand wurde portionenweise in $20 \mathrm{~g}$ eines Gemisches von gleichen Teilen Kalium- und Natriumnitrat eingetragen, das in einer Platinschale zum Schmelzen erhitzt wurde. Nach etwa 15 Minuten entstand eine wasserhelle Schmelze erhalten, die nach dem Erkalten mit $25 \mathrm{ccm}$ konz. Schwefelsäure übergossen und so lange erhitzt wurde, bis alle Salpetersäuredämpfe vertrieben waren, und Schwefelsäuredämpfe auftraten. Die erkaltete Schmelze wurde mit $30 \mathrm{ccm}$ Wasser aufgenommen, quanti-

1) Bei den chemischen Untersuchungen erfreute ich mich der Unterstützung von Dr. Hugo Bau er, Mitglizd des Speyerhauses.

2) Zeitschr. f. anorgan. Chemie 18, 416. 1905.

$\left.{ }^{3}\right)$ Unit. States Departm. of Agriculture. Bureau of Chemistry, Circular Nr. 102.

$\left.{ }^{4}\right)$ Arb. a. d. Kaiserl. Gesundheitsamt 50, 38. 1917.

$\left.{ }^{5}\right)$ Journ. Soc. of Chemic. Ind. 22, 191. 1903. 
tativ in eine Porzellanschale übergespült und durch Zusatz von 10 fach normaler Natronlauge unter Anwendung von Phenolphthalein als Indicator neutralisiert. Die neutrale Lösung wurde in ein Meßkölbchen von $100 \mathrm{com}$ Inhalt übergespült und auf $100 \mathrm{ccm}$ aufgefüllt. $50 \mathrm{ccm} \mathrm{bzw} .25 \mathrm{~cm}$ wurden in eine Glasflasche von $200 \mathrm{ccm}$ gefiullt und mit $100 \mathrm{ccm}$ Normal-Schwefelsäure versetzt. Dann wurden 2 Stückchen Stangenzink von je $3 \mathrm{~cm}$ Länge, die vorher zwecks Erzielung einer gleichmäßigen Wasserstoffentwicklung in einer 1 proz. Kupfersulfatlösung verkupfert wurden, eingeworfen. Das Fläschchen wurde dann sofort mit einem durchbohrten Gummistopfen verschlossen, der 3 hintereinandergeschaltete $\mathrm{Ab}$ sorptionsröhrchen trug. Die beiden der Flasche zunächst liegenden Röhrchen von etwa $1 \mathrm{~cm}$ lichter Weite und $15 \mathrm{~cm}$ Länge waren nach unten verjüngt, mit zusammengerolltem Filtrierpapier gefüllt, das zur Absorption des sich etwa entwickelnden

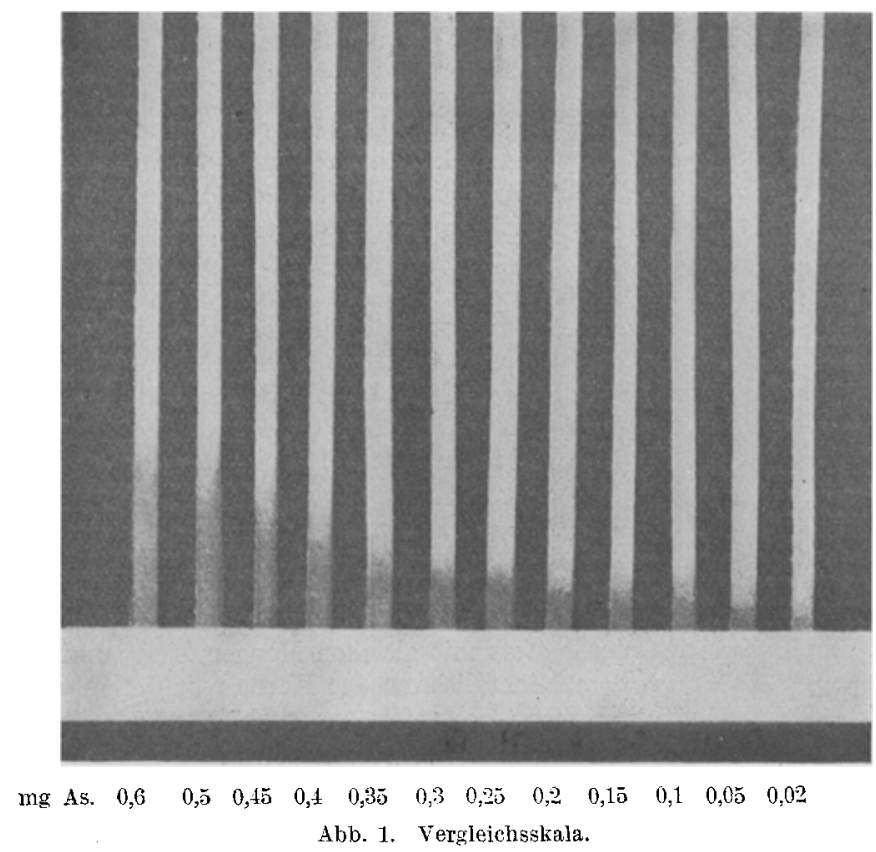

Schwefelwasserstoffs mit Bleiacetatlösung getränkt war. Das 3. Röhrchen von etwa $3 \mathrm{~mm}$ lichter Weite und $15 \mathrm{~cm}$ Länge nahm einen Streifen aus Quecksilberbromidpapier auf, der nach der Vorsobrift von Beck und Merres in folgender Weise hergestellt war: Aus weißem Zeichenpapier wurden Streifen von $20 \mathrm{~cm}$ Länge und $3 \mathrm{~mm}$ Breite geschnitten und 1-2 Stunden in eine 5 proz. alkoholische Lösung von Quecksilberbromid gelegt. Nach dieser Zeit wurden die Streifen herausgenommen, durch leichtes Durchziehen zwischen 2 Fingern von der überschüssigen Lösung befreit und durch kurzes Umschwenken an der Luft getroeknet. Nach Ablauf von 1--2 Stunden war die Entwicklung des Arsenwasserstoffs beendet, und das untere Ende des Streifens hatte eine orangegelbe Farbe angenommen; die Länge des gefärbten Teiles ist der vorhandenen Arsenmenge proportional.

Zur quantitativen Auswertung der Farbstreifen wurden Vergleichsstreifen in der Weise hergestellt, daß normaler Urin mit einer Natriumarsenitlösung von bekanntem Gehalt (von $0,02 \mathrm{mg}$ Arsen steigend bis $0,6 \mathrm{mg}$ ) versetzt und in der 
oben beschriebenen Weise behandelt wurde. Die anf diese Weise erhaltenen Vergleichsstreifen wurden zu einer Skala aneinandergereiht und photographiert, ebenso die bei der Untersuchung der arsenhaltigen Harne erhaltenen Streifen. Die beifolgenden Abbildungen $(1-4)$, die als Beispiele dienen sollen, geben einen guten Über. blick über den Verlauf der Arsenausscheidung durch die Niere.

In den nachstehenden Tabellen, die gleichfalls als Beispiele dienen, sind die bei den einzelnen Arsenobenzolpräparaten erhaltenen Resultate zusammengestellt.

Folgende 3 Kurven mögen zur Demonstration der Ausscheidungsverhältnisse der 3 oben erwähnten Arsenobenzolderivate dienen. Im ganzen wurde der Urin von 20 Patienten in der oben angegebenen Weise in regelmäßigen $\mathrm{Ab}$ ständen untersucht. Die als Beispiel angefuihrten 3 Kurven sind etwa als Mittelwerte zu betrachten. Sie zeigen gewisse prinzipielle Untersehiede, die für diese Prüparate charakteristisch sein dürften und einen gewissen Rüßkschluß auf die Speicherungs- und Ausscheidungsverhältnisse gestatten.

Während die Auss cheidungskurve des Neosalvarsans, wenn man von kleinen Schwankungen absieht, eine anfänglich schwach

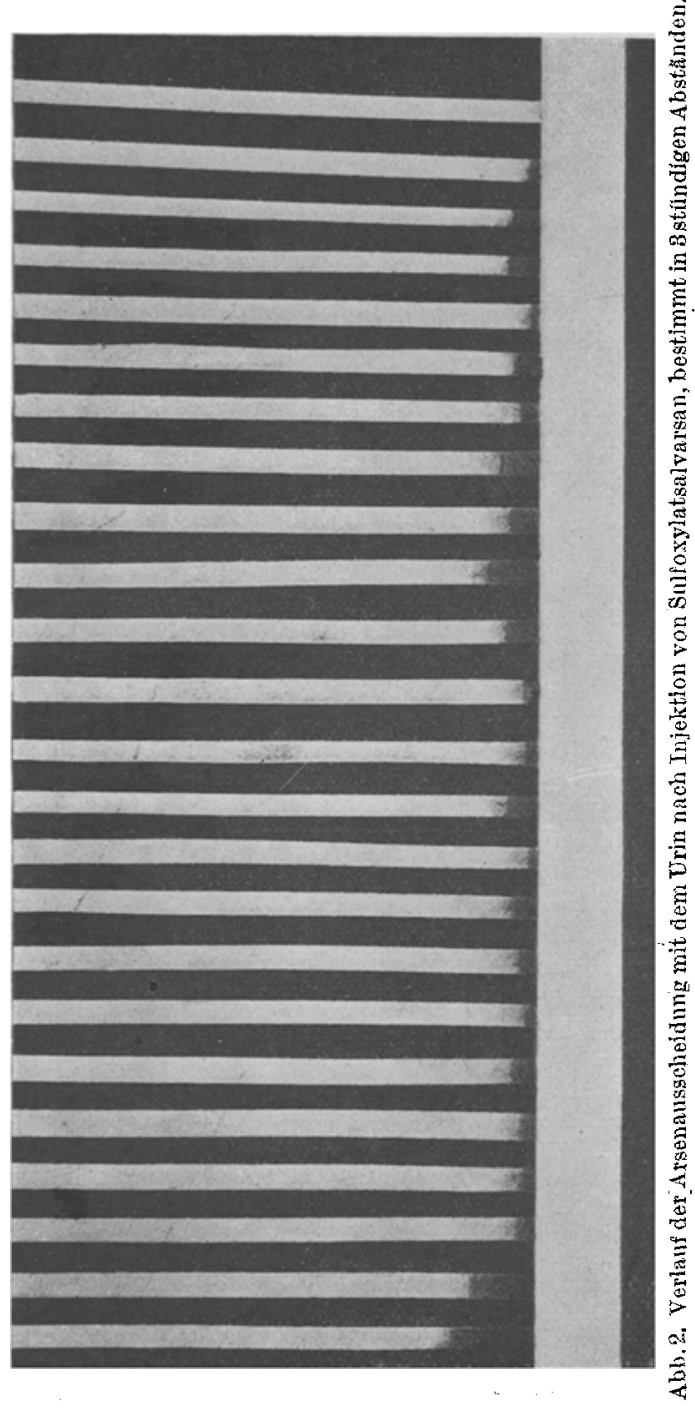
und dann nur ganz allmählich fallende Tendenz aufweist, wird das Silbersalvarsan zunächst in größerer Menge, hiernach aber in Form von richtigen Schüben, unterbrochen von ziemlich regelmäßigen Intervallen, in denen nur ganz geringe Arsenmengen ausgeschieden werden, aus dem Körper eliminiert. Im Gegensatz zum Neo- scheint beim Silbersalvarsan eine Deponierung in Organen, die von 

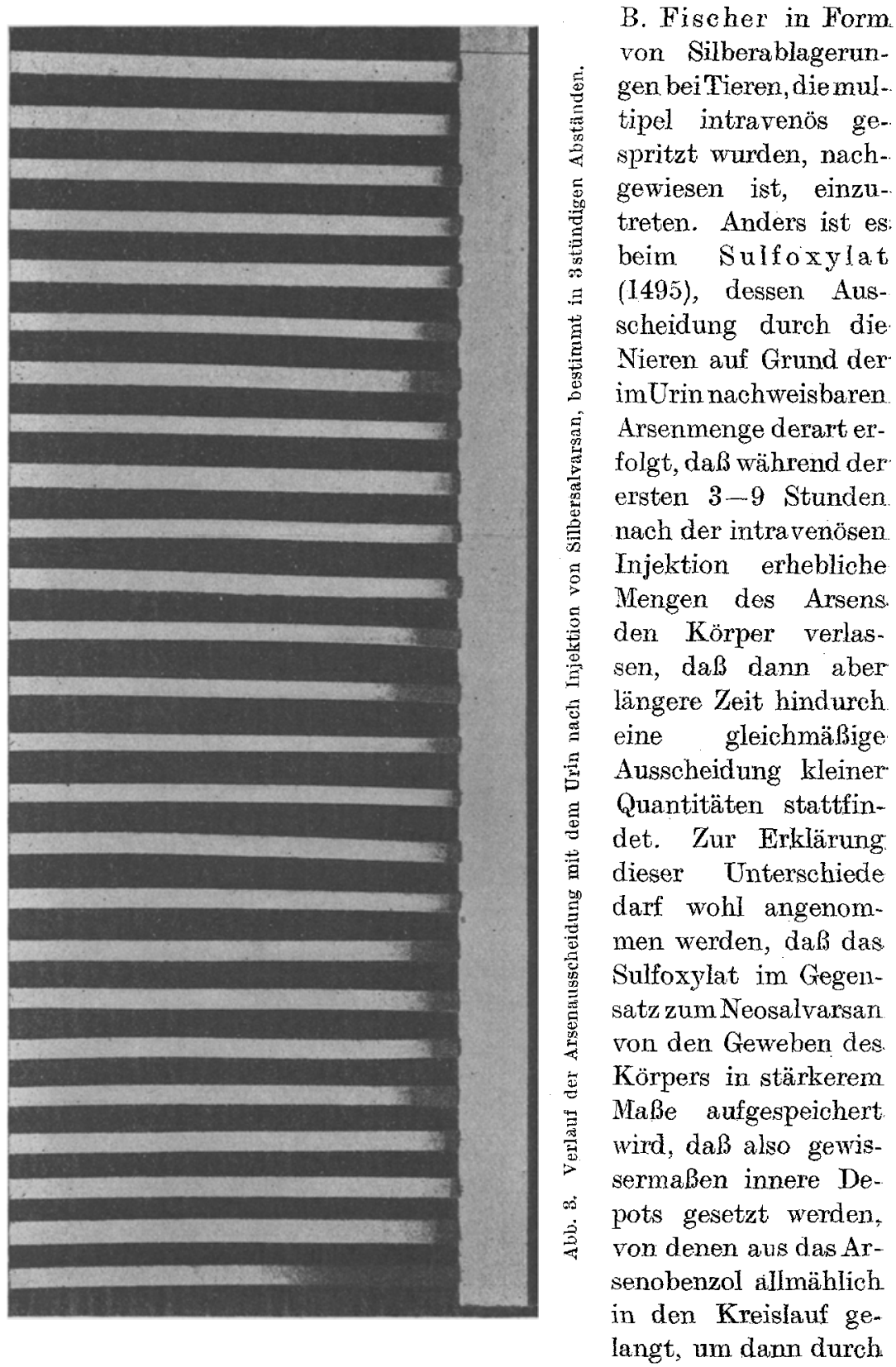

die Nieren ausgeschieden zu werden. Diese Annahme findet ihre Stütze in den Erfahrungen der Kliniker. Aus den Arbeiten von $\mathrm{Nathan}^{1}$ ) and

1) Nathan und Reinecke, Dtsch. med. Wochenschr. 1910, Nr. 1. 
Reinecke, und $\mathrm{Na}$ than und Flehme') geht hervor, daß das Sulfoxylat charakterisiert ist durch die verlangsamte Wirkung, die es auf die floriden Erscheinungen der Syphilis hat. Dafür sprach neben der Langsamkeit noch die Gleichmäßigkeit der Rückbildung der floriden Erscheinungen, das Ausbleiben von bald nach der Injektion auftretender Herxheimerscher Reaktion und die sog. ,Spätreaktion" am vierten Tage nach der Injektion. Diese lange Remanenz und langsamere, jedoch lang. anhaltende Wirkung INathan und Reineckel.c., W.Key ${ }^{2}$, W. Sasse ${ }^{3}$ ), G. Fant14), R. Neuen dorf5), W. Arnol1) Nathan und Fleh me, Therap. Halbmonatshefte 34, H. 21.

2)W.Key, UberSulfoxylatsalvarsan. Inaug.Diss. Gießen 1919.

3) W. Sasse, Über Silbersalvarsan und Sulf. oxylat. Inaugural-Diss. Gießen 1919.

4) G. Fantl, Dermatol. Wochenschr. ro, 155. 1920.

5) R. Nevendorf, Dermatol. Wochenschr. r1, 883. 1920 .

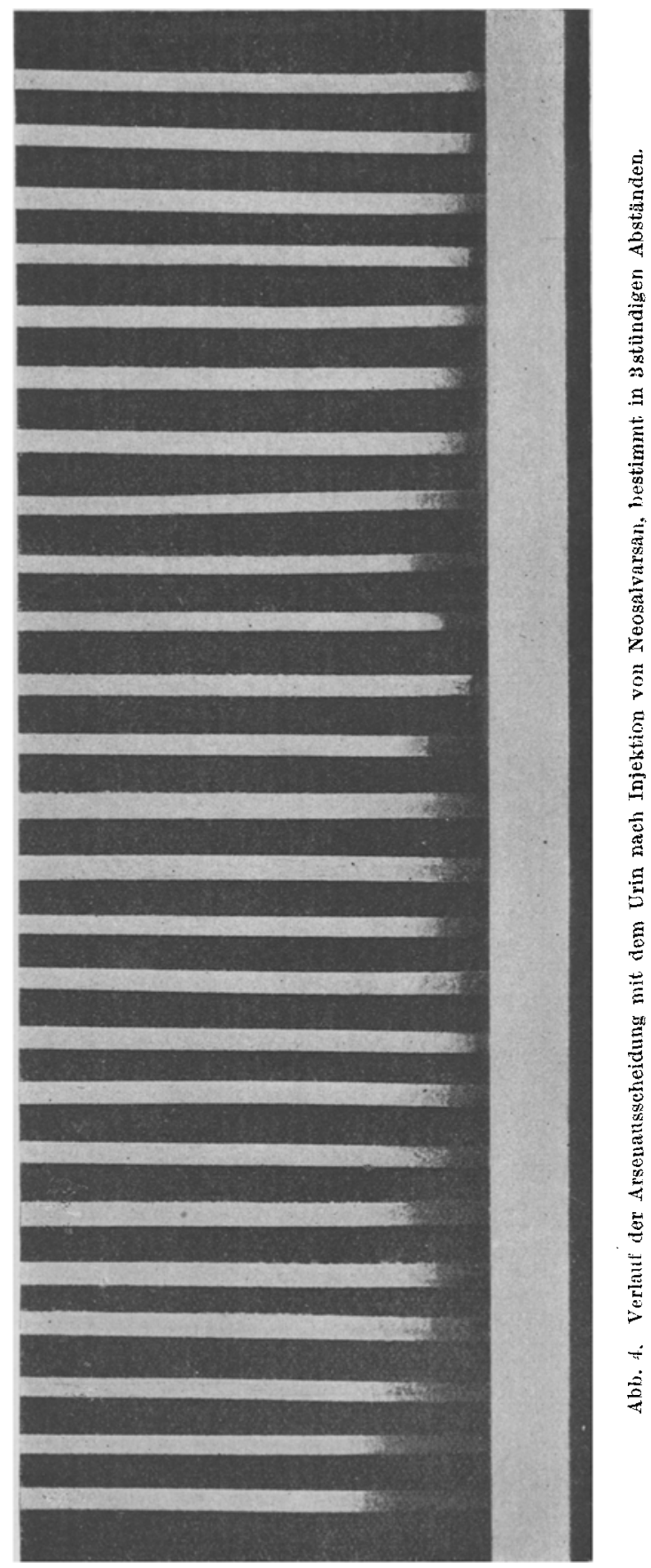


di $i^{1}$ ), J. Fabr $\left.\mathrm{y}^{2}\right)$ ], die zwar den chemo-therapeutischen Ictus verschieben kann, hat aber gegenïber dem nur kurze Zeit im Körper kreisenden Neosalvarsan den großen Vorteil, daß der Organismus längere Zeit. hindurch mit dem Arsenobenzolderivat gewissermaßen vollständig durchtränkt wird. Die Möglichkeit, sämtliche im Gewebe versteckten Treponemen abzutöten, ist dadurch wesentlich erhöht.

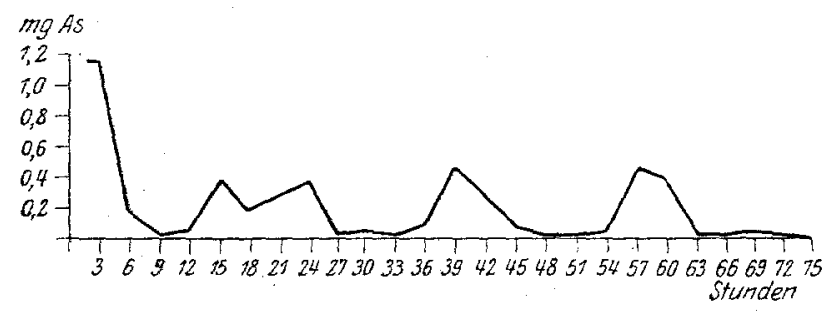

Abb. 5. Silbersalvarsan.

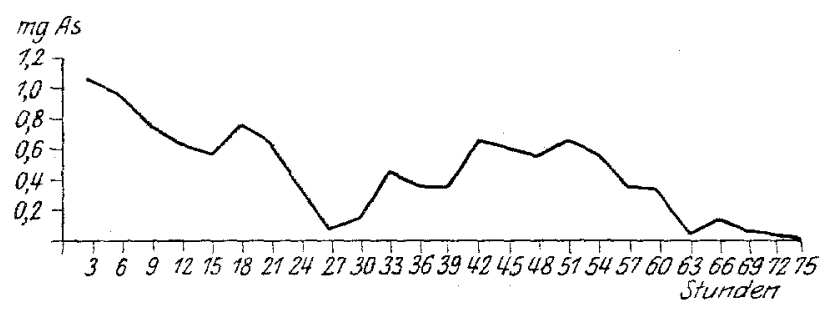

Abb. 6. Neosalvarsan.

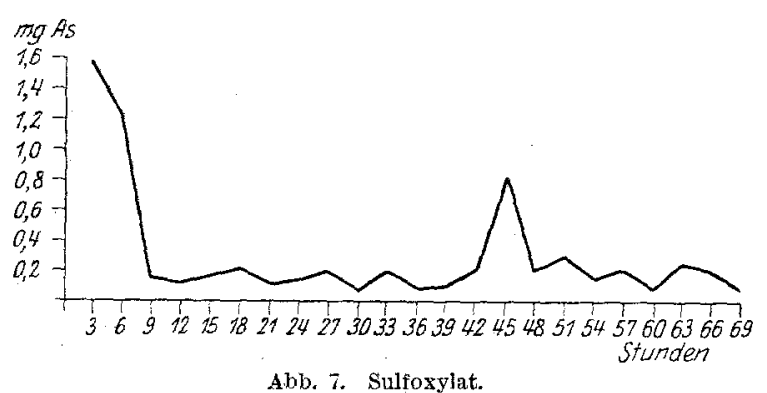

Verlauf der Arsenausscheidung mit dem Urin nach Injektion von Arsenobenzolderivaten, bestimmt in 3 stiondigen Abständen.

Da das Sulfoxylatsalvarsan im. Gegensatz zum Neosalvarsan gegen Oxydationseinflüsse beständig und in Lösung stabil ist, ist zu erwarten, daß es auch im Organismus längere Zeit in unveränderter Form gespeichert wird. Von diesem Gesichtspunkte aus erscheint das Sulfoxylat für eine länger dauernde Durchtränkung der Säfte des Organismus mit einem wirksamen Arsenobenzolderivat besonders geeignet.

1) W. Arnoldi, Berl. klin. Wochenschr. 1921, Nr. 2, S. 29.

2) J. Fabry, Med. Klin. 1921, Nr. 27. 
Neosalvarsan, Sulfoxylatsalvarsan und Silbersalvarsan durch die Niere usw. 63

Versuch XVIII. Sulfoxylat $0,2=0,04$ Arsen.

\begin{tabular}{|c|c|c|c|}
\hline $\begin{array}{l}\text { Anzahl der } \\
\text { Stunden nach } \\
\text { der Injektion }\end{array}$ & $\begin{array}{c}\text { Ausgeschiedene } \\
\text { Harnmenge inner- } \\
\text { halb } 3 \text { Stunden } \\
\text { ccm }\end{array}$ & $\begin{array}{c}\text { Arsen } \\
\text { gefunden } \\
\text { mg }\end{array}$ & $\begin{array}{l}\text { Im Liter } \\
\text { Arsen } \\
\text { gefunden } \\
\text { mg }\end{array}$ \\
\hline 3 & 150 & 1,6 & 10,7 \\
\hline 6 & 200 & 1,2 & 6,0 \\
\hline 9 & 140 & 0,1 & 0,71 \\
\hline 12 & 150 & 0,1 & 0,67 \\
\hline 15 & 60 & 0,15 & 2,5 \\
\hline 18 & 100 & 0,2 & 2,0 \\
\hline 21 & 110 & 0,08 & 0,72 \\
\hline 24 & 180 & 0,1 & 0,6 \\
\hline 27 & 200 & 0,2 & 1,0 \\
\hline 30 & 125 & 0,05 & 0,4 \\
\hline 33 & 220 & 0,2 & 0,9 \\
\hline 36 & 180 & 0,1 & 0,6 \\
\hline 39 & 125 & 0,1 & 0,8 \\
\hline 42 & 225 & 0,2 & 0,9 \\
\hline 45 & 200 & 0,8 & 4,0 \\
\hline 48 & 180 & 0,2 & 1,1 \\
\hline 51 & 140 & 0,3 & 2,1 \\
\hline 54 & 150 & 0,15 & 1,0 \\
\hline 57 & 225 & 0,2 & 0,9 \\
\hline 60 & 200 & 0,05 & 0,25 \\
\hline 63 & 180 & 0,25 & 1,4 \\
\hline 66 & 200 & 0,2 & 1,0 \\
\hline 39 & & 0,05 & \\
\hline Versuoh & XIX. $\quad 0,2 \mathrm{~g}$ Si & alvarsan & Arsen. \\
\hline 3 & 280 & 1,2 & 4,3 \\
\hline 6 & 230 & 0,2 & 0,87 \\
\hline 9 & 250 & 0,02 & 0,88 \\
\hline 12 & 220 & 0,05 & 0,23 \\
\hline 15 & 200 & 0,4 & 2,0 \\
\hline 18 & 140 & 0,2 & 1,4 \\
\hline 21 & 90 & 0,3 & 3,3 \\
\hline 24 & 125 & 0,4 & 3,2 \\
\hline 27 & 285 & 0,02 & 0,07 \\
\hline 30 & 90 & 0,04 & 0,44 \\
\hline 33 & 130 & 0,02 & 0,15 \\
\hline 36 & 200 & 0,1 & 0,5 \\
\hline 39 & 170 & 0,5 & 2,9 \\
\hline 42 & 125 & 0,3 & 2,4 \\
\hline 45 & 130 & 0,1 & 0,77 \\
\hline 48 & 200 & 0,05 & 0,25 \\
\hline$\check{a 1}$ & 320 & 0,05 & 0,16 \\
\hline 54 & 180 & 0,07 & 0,39 \\
\hline 57 & 225 & 0,5 & 2,22 \\
\hline 60 & 200 & 0,4 & 2,0 \\
\hline 63 & 200 & 0,05 & 0,25 \\
\hline 66 & 140 & 0,05 & 0,36 \\
\hline 69 & 200 & 0,1 & 0,5 \\
\hline 72 & 200 & 0,05 & 0,25 \\
\hline 75 & 180 & 0,02 & 0,11 \\
\hline
\end{tabular}


64 E. Bernstein: Vergleichende Untersuchungen aber die Ausscheidnng usw.

Versuch XX. Neo-Dosis $\mathrm{II}=0,3 \mathrm{~g}=0,06$ Arsen.

$\begin{array}{cccc}\begin{array}{c}\text { Anzahl der } \\ \text { Stunden nach } \\ \text { der Injektion }\end{array} & \begin{array}{c}\text { Ausgeschiedene } \\ \text { Harnmenge inner- } \\ \text { halb 3 Stunden } \\ \text { cem }\end{array} & \begin{array}{c}\text { Arsen } \\ \text { gefunden }\end{array} & \begin{array}{c}\text { Im Liter } \\ \text { Arsen } \\ \text { gefunden } \\ \text { mg }\end{array} \\ 3 & 200 & 1,1 & 5,5 \\ 6 & 220 & 1,0 & 4,5 \\ 9 & 320 & 0,8 & 2,5 \\ 12 & 185 & 0,7 & 3,8 \\ 15 & 180 & 0,6 & 3,3 \\ 18 & 300 & 0,8 & 2,7 \\ 21 & 100 & 0,7 & 7,0 \\ 24 & 250 & 0,4 & 1,6 \\ 27 & 290 & 0,1 & 3,4 \\ 30 & 350 & 0,2 & 0,6 \\ 33 & 260 & 0,5 & 1,9 \\ 36 & 280 & 0,4 & 1,4 \\ 39 & 360 & 0,4 & 1,1 \\ 42 & 320 & 0,7 & 2,2 \\ 45 & 290 & 0,1 & 3,4 \\ 48 & 280 & 0,6 & 2,1 \\ 51 & 300 & 0,7 & 2,3 \\ 54 & 270 & 0,6 & 2,2 \\ 57 & 260 & 0,4 & 1,5 \\ 60 & 175 & 0,4 & 2,3 \\ 63 & 200 & 0,1 & 0,5 \\ 66 & 290 & 0,2 & 0,7 \\ 69 & 200 & 0,1 & 0,5 \\ 72 & 125 & 0,05 & 0,4 \\ 75 & 100 & 0,05 & 0,5\end{array}$

